Dans ce travail, j'ai cherché à montrer comment sont dirigés les efforts, et pourquoi, en cette matière, tous les intérêts généraux en jeu sont convergents. Il m'apparaît donc que le problème posé peut être résolu, malgré les difficultés; qui, si elles existent réellement, ne sont pas insurmontables.

\title{
PRÉSURES VÉGÉTALES. EXTRACTION ET PROPRIÉTÉS \\ par
}

\section{CHRISTEN et E. VIRASORO}

(Institut de Recherches 'Scientifiques et Techniques de la Faculté de Chimie Industrielle. Université Nat. du Litoralle, Santa-Fé, R. Argentine. Directeur : Dr. H. Damianovich.)

$$
\text { (Fin) }
$$

Si l'on compare les courbes citées avec la figure $n^{0} 3$, qui traduit les résultats des expériences de Fleischmann [5] faites avec la présure animale, on voit dans l'une aussi bien que dans l'autre, que les temps diminuent progressivement jusqu'à atteindre un minimum

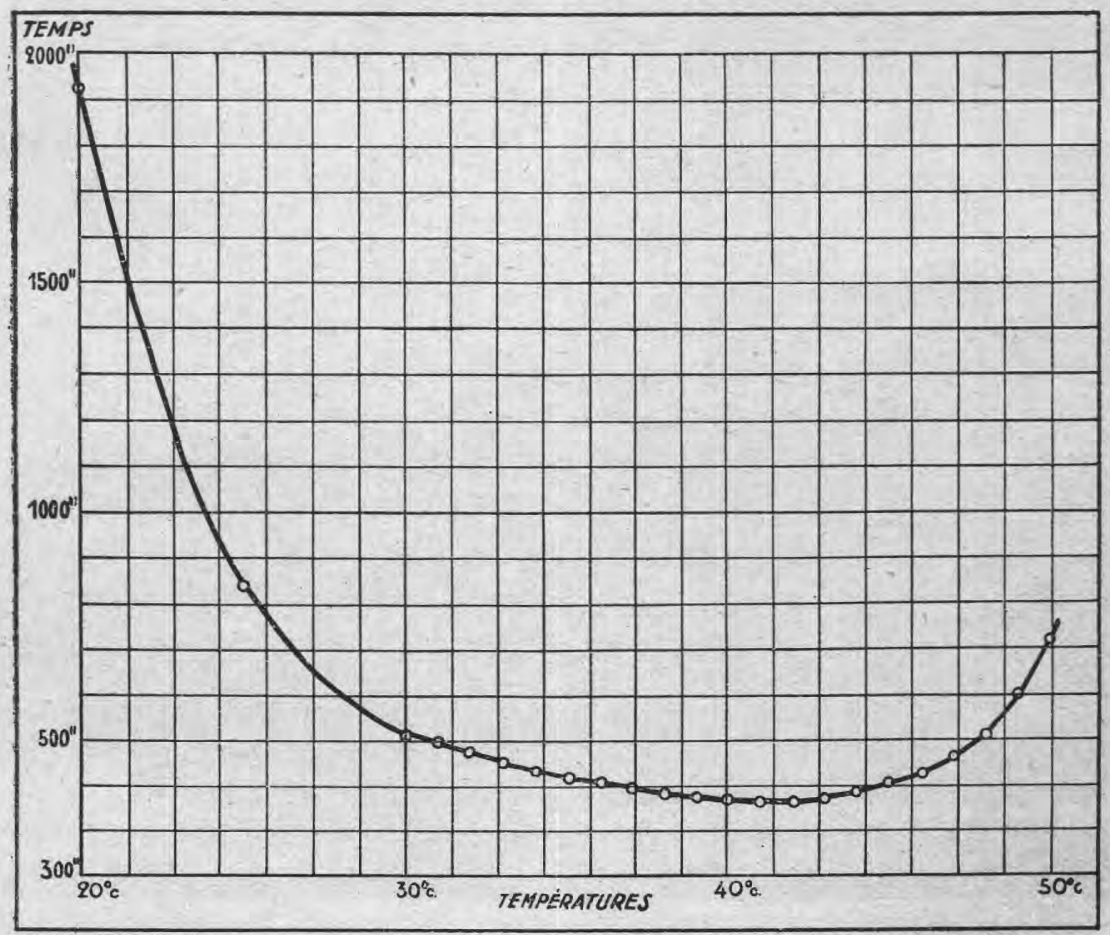

FigURE 3. 
- pour la présure végétale, $68^{\circ} \mathrm{C}$, et pour l'animale, $41^{\circ} \mathrm{C}$. Cependant, tandis qu'avec la présure animale les temps de coagulation augmentent progressivement à partir de l'optima, cette partie de la courbe ayant une allure semblable à la première partie, avec la présure végétale la courbe montre une ascension rapide du temps, si rapide que $2-3^{\circ}$ après l'optima, la vitesse de coagulation est pratiquement nulle.

On peut considérer que dans ce phénomène il y a deux actions superposées :

a) A mesure que la température s'élève, l'ionisation des électrolites dans le lait augmente, c'est-à-dire que la vitesse de la réaction enzymatique croît (influence des ions $H$ et $\mathrm{Ca}$ ) jusqu'à un point limité par la température à laquelle l'insolubilisation des sels de calcium commence à se produire.

b) L'inactivation graduelle que subit la présure par suite de l'élévation progressive de la température, comme on le voit, cidessous :

Présure végétale d'activité 1 : 350 ,

Présure végétale d'activité 1 : 480, chauffée pendant 25 minutes chauffée pendant á minutes

\begin{tabular}{l|c||c|c}
\hline \hline Températures & Temps de coagulation & Températures & Temps de coagulation \\
\cline { 2 - 4 } Témoin & $345^{\prime \prime}$ & Témoin & $250^{\prime \prime}$ \\
Chauffée à $40^{\circ}$ & $406^{\prime \prime}$ & Chauffée à $40^{\circ}$ & $247^{\prime \prime}$ \\
Chauffée à $45^{\circ}$ & $414^{\prime \prime}$ & Chauffée à $50^{\circ}$ & $248^{\prime \prime}$ \\
Chauffée à $50^{\circ}$ & $462^{\prime \prime}$ & Chauffée à $55^{\circ}$ & $250^{\prime \prime}$ \\
Chauffée à $55^{\circ}$ & $577^{\prime \prime}$ & Chauffée à $60^{\circ}$ & $275^{\prime \prime}$ \\
Chauffée à $60^{\circ}$ & $1.206^{\prime \prime}$ & Chauffée à $65^{\circ}$ & $525^{\prime \prime}$ \\
Chauffée à $65^{\circ}$ & pas de coagulation en & Chauffée à $70^{\circ}$ & $1.190^{\prime \prime}$ \\
& $3.000^{\prime \prime}$ & Chauffée à $75^{\circ}$ & pas de coagulation en \\
& & & $2.700^{\prime \prime}$ \\
\hline \hline
\end{tabular}

Dans ces essais, on a chauffé, pendant 5 à 25 minutes, des solu. tions de présure végétale de même activité, aux températures indiquées dans le tableau, on les a refroidies tout de suite, en mesurant aussitôt après, dans les mêmes conditions, leur activité à $35^{\circ}$.

Nous croyons qu'il faut tenir compte que la coagulation de la lacto-albumine se fait justement à la température à laquelle s'observe la chute de l'activité. Cette perte si rapide pourrait-elle être même due à une disparition de la présure par un emprisonnement dans l'albumine insolubilisée ?

D'après PARISI [6] la température d'inactivation de cette présure serait comprise entre $65^{\circ}$ et $70^{\circ} \mathrm{C}$. A notre avis, on ne doit pas parler, dans ce cas, d'une température d'inactivation car le temps de 
caléfaction est un facteur qui agit considérablement dans la destruction de cette enzyme.

Par exemple, à $50^{\circ}$ on pourrait inactiver complètement la présure ; seulement il faut la chauffer pendant un temps suffisamment prolongé. Cependant on ne peut pas dire dans ce cas, que la température d'inactivation de cette présure se trouve près de $50^{\circ}$. D'ailleurs, la résistance de la présure à la ehaleur est, comme nous l'avons déjà vi, facteur du degré de sa dilution.

\section{Loi de Segelcke-Storch.}

Une des propriétés les plus caractéristiques des présures est de suivre avec une certaine approximation, la loi de Segelcke-Storch, d'après laquelle, le produit du temps de coagulation du lait par la concentration en enzyme est constant :

$$
\mathrm{t} . \mathrm{c}=\mathrm{k}
$$

Nombre d'auteurs se sont employés à vérifier cette loi avec la présure animale et ces résultats sont vraiment très discordants entre eux.

Dans une étude sur cette loi [7], nous avions observé que dans la coagulation du lait, le produit "temps $\times$ concentration " diminuait légèrement avec les temps. Par contre, avec le complexe "caséinate de Ca-phosphate de Ca", composant du lait qui subit l'action enzymatique, nous avions trouvé que ce produit augmentait sensiblement avec les temps.

Nous avons jugé intéressante l'étude du comportement de la présure végétale vis-à-vis de cette loi, ce qui nous permettrait en même temps de comparer les caractéristiques des deux présures.

Les résultats des essais de vérification de cette loi avec la présure du chardon, à $35^{\circ}$ et $50^{\circ}$, sont indiqués dans le tableau suivant :

è $35^{\circ}$

à $50^{\circ}$

\begin{tabular}{l|c|c||c|c|c}
\hline \hline $\begin{array}{c}\text { Volume de } \\
\text { présure pour cent } \\
\text { de lait }\end{array}$ & $\begin{array}{c}\text { Temps de } \\
\text { coagulation }\end{array}$ & $\begin{array}{c}\text { Produit : } \\
\text { Cone. } \times \\
\text { temps }\end{array}$ & $\begin{array}{c}\text { Volume de } \\
\text { présure pour cent } \\
\text { de lait }\end{array}$ & $\begin{array}{c}\text { Temps de } \\
\text { coagulation }\end{array}$ & $\begin{array}{c}\text { Produit: } \\
\text { Cone. } \times \\
\text { temps }\end{array}$ \\
\cline { 2 - 4 } $4 \mathrm{~cm}^{3}$ & $229^{\prime \prime}$ & 916 & $3 \mathrm{~cm}^{3}$ & $193^{\prime \prime}$ & 579 \\
$3 \mathrm{~cm}^{3}$ & $287^{\prime \prime}$ & 861 & $2 \mathrm{~cm}^{3}$ & $295^{\prime \prime}$ & 591 \\
$2 \mathrm{~cm}^{3}$ & $398^{\prime \prime}$ & 796 & $1,333 \mathrm{~cm}^{3}$ & $451^{\prime \prime}$ & 601 \\
$1,332 \mathrm{~cm}^{3}$ & $576^{\prime \prime}$ & 767 & $1 \mathrm{~cm}^{3}$ & $616^{\prime \prime}$ & 616 \\
$1 \mathrm{~cm}^{3}$ & $735^{\prime \prime}$ & 735 & $0,666 \mathrm{~cm}^{3}$ & $980^{\prime \prime}$ & 653 \\
$0,666 \mathrm{~cm}^{3}$ & $1.122^{\prime \prime}$ & 747 & $0,5 \mathrm{~cm}^{3}$ & $1.505^{\prime \prime}$ & 752 \\
$0,5 \mathrm{~cm}^{3}$ & $1.498^{\prime \prime}$ & 749 & $0,4 \mathrm{~cm}^{3}$ & $1.867^{\prime \prime}$ & 747 \\
$0,4 \mathrm{~cm}^{3}$ & $1.950^{\prime \prime}$ & 780 & $0,333 \mathrm{~cm}^{3}$ & $2.660^{\prime \prime}$ & 886 \\
$0,333 \mathrm{~cm}^{3}$ & $2.469^{\prime \prime}$ & 822 & 0,286 & $3.250^{\prime \prime}$ & 926 \\
$0,286 \mathrm{~cm}^{3}$ & $2.820^{\prime \prime}$ & 805 & & & \\
\hline \hline
\end{tabular}


En analysant les courbes 1 et 11 (figure no 4 ) représentant la fonction temps-produit de ces expériences, aux températures de $35^{\circ}$ et $50^{\circ}$ respectivement, on peut observer dans la première une descente jusqu'aux 12 minutes et dès lors une montée progressive ; tandis que la deuxième ne présente pas la chute initiale mais une ascension plus rapide.

On voit done que l'agent qui a fait retarder la vitesse de la réaction à $35^{\circ}$ s'est fait sentir avec plus d'intensité à $50^{\circ}$. Pour expliquer ces anomalies nous considérons qu'il est nécessaire d'analyser les points suivants :

$1^{\circ}$ Influence de la ehaleur sur la présure, à la température de l'expérience.

$2^{\circ}$ Influence de la dilution de la présure.

$3^{\circ}$ Développement de la fermentation dans le lait pendant l'emprésurage.

$4^{\circ}$ Erreur dans la mesure du temps de coagulation.

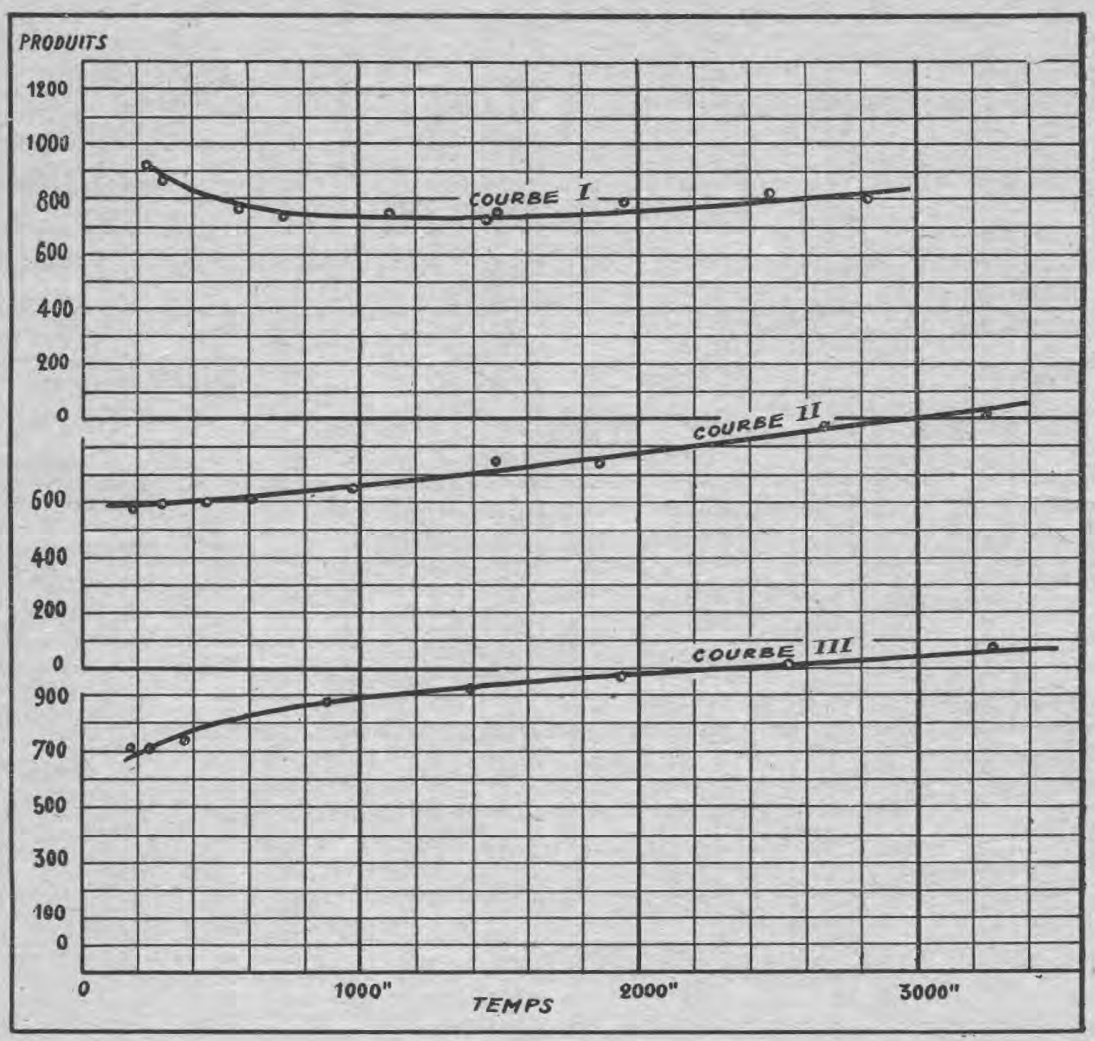

Figure 4. 
En étudiant chacun de ces facteurs, nous verrons que les uns ont un effet accélérateur dans la coagulation, tandis que les autres, au contraire, retardent le travail de la présure.

10 influence de la chaleur sur la présure. - Si l'on chauffe dans un bain-marie une solution de présure animale à $40^{\circ}$ et si, à des intervalles de temps, on prélève des échantillons, en mesurant ensuite leur activité dans les mêmes conditions (température de coagulation, volumes de lait et de présure), on observe que l'activité de cette solution diminue progressivement avec le temps de caléfaction. C'est ce qu'on constate dans le tableau suivant :

\begin{tabular}{c|c}
\hline \hline $\begin{array}{c}\text { Temps d'échauffement } \\
\text { à } 40^{\circ}\end{array}$ & Temps de coagulation \\
\hline & \\
Témoin & $232^{\prime \prime}$ \\
$5^{\prime}$ & $287^{\prime \prime}$ \\
$10^{\prime}$ & $325^{\prime \prime}$ \\
$15^{\prime}$ & $347^{\prime \prime}$ \\
$20^{\prime}$ & $360^{\prime \prime}$ \\
$25^{\prime}$ & $385^{\prime \prime}$ \\
$30^{\prime}$ & $440^{\prime \prime}$ \\
& \\
\hline \hline
\end{tabular}

La présure végétale est aussi sensible à l'action de la chaleur ; ainsi, à la température correspondante à l'essai représenté par la courbe 1 (à $35^{\circ}$ ), l'inactivation est peu visible, mais à $50^{\circ}$ (courbe 11) l'effet de la chaleur est plus notable : une solution 1 : 700 perd, au bout de 25 minutes, $26 \%$ de son activité.

$2^{\circ}$ influence de LA Dilution. - Au cours des essais de vérification de la loi citée, la concentration de la présure dans le lait a varié de 1 à 15. Ces variations doivent se faire sentir sans doute, comme nous l'avons déjà vu.

$3^{\circ}$ SELF-ACIDIFICATION du LAit. - Nous savons qu'à la température de $35^{\circ}$ le développement des ferments lactiques est à son maximum. Si l'on porte du lait, emprésuré ou non, à cette température, la self-acidification due aux ferments lactiques sera accélérée, par conséquent, la concentration en ions $H$ augmentera, e'est-à-dire que ce lait sera coagulé par la présure dans un temps plus court.

Un échantillon de lait bien conservé est placé dans un bainmarie pendant une heure :

Temps de coagulation avant l'essai .............. $465^{\prime \prime}$

Temps de coagulation a près l'essai $\ldots \ldots \ldots \ldots \ldots \ldots \ldots .378^{\prime \prime}$

ce qui représente une avance dans la coagulation égale à $19 \%$ du temps initial. 
Malheureusement, on ne peut pas stériliser préalablement le lait pour éviter cette acidification, puisque cela provoquerait un trouble dans sa composition. De même on ne peut pas arrêter le travail des ferments au moyen des antiseptiques, la plupart d'entre eux anéantissant la présure.

$4^{\circ}$ ERREUR dANS LA mesure de LA coAgulation. - Le phénomène de la coagulation du lait par la présure n'est pas encóre assez connu. Nous ne savons pas exactement si le commencement de la coagulation nous indique précisément la fin de la réaction enzymatique, c'est-à-dire la transformation quantitative du caséinate en para-caséinate.

Dans les emprésurages de courte durée, une petite différence dans sa mesure produira des erreurs négligeables, mais on comprend que dans les longs emprésurages cette erreur augmentera fortement.

$$
*^{*} *
$$

Afin d'éliminer les facteurs propres au lait qui peuvent agir sur la coagulation (self-acidification, albumines, etc.), nous avons essayé la vérification de la loi de Segelcke-Storch avee le complexe caséinate de Ca-phosphate de Ca. Voici les résultats pour la température de $35^{\circ}$ :

\begin{tabular}{c|c|c}
\hline $\begin{array}{c}\text { Volume de présure pour } 100 \mathrm{~cm}^{3} \\
\text { de complexe }\end{array}$ & $\begin{array}{c}\text { Temps de coagula- } \\
\text { tion en secondes }\end{array}$ & Produit : concentr. $\times$ temps \\
\hline $4 \mathrm{~cm}^{3}$ & 179 & 716 \\
3 & 234 & 702 \\
2 & 371 & 742 \\
1 & 882 & 882 \\
0,666 & 1.398 & 931 \\
0,5 & 1.938 & 969 \\
0,4 & 2.528 & 1.011 \\
0,333 & 3.280 & 1.092 \\
\hline
\end{tabular}

En comparant l'allure de la courbe 111 (figure $n^{\circ} 3$ ), où sont représentés les résultats de cette expérience, avec celle de la courbe 1 qui correspond à la même expérience, mais faite avec le lait, on voit que le produit "temps $x$ concentration" augmente un peu plus lentement avec le temps dans la coagulation du complexe que dans celle du lait, ee qui peut s'expliquer par l'absence du lactose et des ferments lactiques dans celui-là, lesquels, comme on vient de le voir, provoquent une accélération de la coagulation.

$$
\text { *** }
$$


La coagulation du lait par les présures en général devrait suivre rigoureusement, à notre avis, la loi de Segeleke-Storch. Si cela ne s'accomplit pas, c'est à cause des facteurs étrangers au phénomène même de la coagulation, facteurs que nous avons déjà énumérés.

\section{INFLUENCE DES BASSES TEMPERATURES}

Le comportement des enzymes soumises à des basses températures n'est pas encore très connu.

HALDANe [8] dit à cet égard : "nombre d'enzymes ne sont pas détruites rapidement à la température de l'air liquiden; d'où on déduit, qu'en général, les enzymes doivent subir une diminution d'activité, quand on les refroidit à la température de l'air liquide.

D'après nos expériences, nous pouvons conclure que les deux présures, animale et végétale, ne perdent pas absolument leur activité lors d'un refroidissement dans l'air liquide; il semble au contraire qu'il y ait activation de leur pouvoir coagulant.

Des solutions de présures (de caillettes de veau et de chardon), d'activité à peu près équivalente, ont été mises dans des tubes à essai, bien bouchés, et refroidies à la température de $-180^{\circ}$, pendant 2 à 3 heures $1 / 2$. Une fois revenues à la température du laboratoire, les deux solutions sont limpides.

\begin{tabular}{c|c|c}
\hline \multicolumn{1}{c|}{ Présure } & \multicolumn{2}{|c}{ Temps de coagulation } \\
\cline { 2 - 3 } & Témoin & Refroidie \\
\hline Présure végétale $(1: 350) \ldots \ldots \ldots \ldots$ & $682^{\prime \prime}$ & $640^{\prime \prime}$ \\
Présure animale $(1: 250) \ldots \ldots \ldots \ldots$ & $950^{\prime \prime}$ & $791^{\prime \prime}$ \\
\hline \hline
\end{tabular}

Dans chaque essai : $0 \mathrm{~cm}^{3}$ de solution-présure dans $10 \mathrm{~cm}^{3}$ de lait, à $35^{\circ}$.

Ainsi, une solution de présure végétale d'une activité $1: 350$, soumise pendant 3 heures à eette très basse température, augmente son pouvoir coagulant de $6 \%$ à peu près. Avec les solutions de présure animale d'une activité $1: 250$, et également refroidies, cette activation est plus considérable encore ; elle équivaut à $17 \%$.

Probablement, cette activation observée est due à la destruction, aux basses températures, des anti-enzymes.

\section{EFFET DES RADIATIONS}

L'action destructive des radiations visibles et des rayons ultraviolets sur les enzymes est bien connue; cependant HaLdaNe [9] a fait remarquer une action activante. 
Nous avons étudié l'effet de ces radiations, tant sur la présure du chardon que sur celle de caillette de veau.

\section{Radiations visibles.}

Des solutions étendues des présures citées d'à peu près la même activité (1:200), mises dans des tubes en verre afin d'éliminer les rayons ultra-violets, sont exposées aux rayons solaires pendant deux heures (de 16 à 18 heures au commencement d'automne).

Les activités de la présure exposée et du témoin sont indiquées dans le tableau suivant :

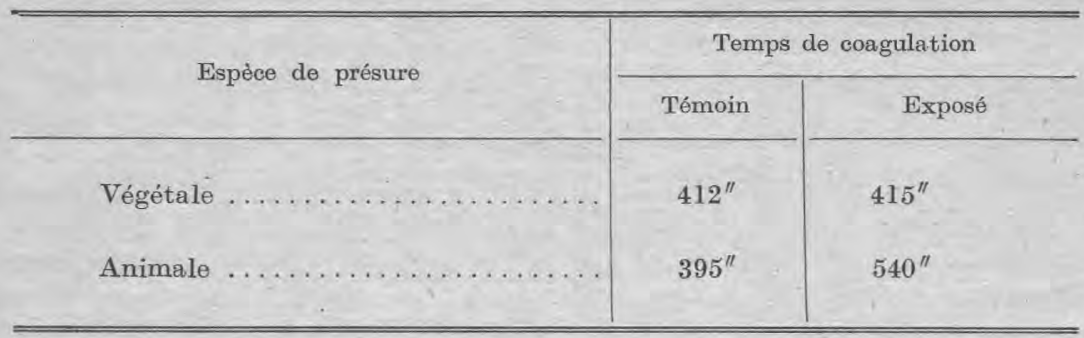

Tandis que la solution de présure végétale n'a pas perdu de son activité, après avoir été exposée aux rayons solaires, la présure animale a perdu $27 \%$ de son pouvoir coagulant. Nous faisons remarquer que la solution de présure du chardon possédait une très légère teinte caramel.

\section{Rayons ultra-violets.}

Ces mêmes solutions de présures sont exposées dans des tubes en quartz, aux rayons ultra-violets. La source de rayons ultraviolets est constituée par un arc électrique entre deux électrodes en fer, d'une tension de 15.000 volts. Les échantillons ont été placés à $10 \mathrm{~cm}$. pendant une heure.

\begin{tabular}{|c|c|c|}
\hline \multirow{2}{*}{ Espèce de présure } & \multicolumn{2}{|c|}{ Temps de coagulation } \\
\hline & Témoin & Exposé \\
\hline Végétale ........... & $412^{\prime \prime}$ & $417^{\prime \prime}$ \\
\hline Animale $\ldots \ldots \ldots \ldots \ldots \ldots \ldots$ & $395^{\prime \prime}$ & $2.190^{\prime \prime}$ \\
\hline
\end{tabular}

Comme dans l'expérience antérieure, la présure végétale n'a pas changé dans son activité (la petite différence observée peut être considérée comme une erreur d'essai). La présure animale, par contre, a vu son activité réduite à $18 \%$ de sa valeur primitive. 
Ces valeurs ne sont que relatives; la sensibilité des enzymes aux radiations varie avec la concentration, puisqu'à une plus grande dilution, plus grand est aussi l'effet destructif. Il arrive de même selon le degré de purification : on sait que les substances étrangères qui accompagnent les enzymes semblent les protéger de l'effet des radiations.

$\mathrm{Si}$ nous considérons que les présures animales sont originaires d'un milieu où elles ne sont pas atteintes par les radiations visibles et les rayons ultra-violets, nous comprenons qu'elles soient plus sensibles à ces radiations que celles d'origine végétale, qui ont cependant des propriétés très semblables mais qui sont constamment exposées aux rayons solaires.

\section{ESSAIS TECHNIQUES}

Afin d'étudier les possibilités de son emploi dans l'industrie fromagère, nous avons réalisé des essais de fabrication de fromages avec cette présure végétale (1). C'est le camembert qui a été choisi pour ces essais, parce qu'il est d'une classe de fromages dont la fabrication est des plus délicates; de cette façon, cette présure était donc soumise à une épreuve rigoureuse.

Pour obtenir des résultats comparatifs, nous avons exécuté deux essais, parallèlement, avec la présure du chardon et une présure animale du commerce (en poudre), en nous plaçant dans les mêmes conditions (emprésurage, mise en moule, salage, soins en cave, etc.).

Les fromages préparés avec la présure du chardon atteignirent leur maturation quelques jours avant ceux fabriqués à l'aide de la présure animale. Tant par l'aspect de la pâte que par son goût, les premiers ont été considérés aussi bons que les témoins (présure animale). Dans les premiers jours de maturation, les fromages de présure du chardon présentaient un goût légèrement astringent qui disparut quand la maturation arriva à sa fin. Ce goût provient du tanin et de diverses autres substances qui passent en solution pendant la macération des fleurs; elles peuvent être, d'ailleurs, si on le désire, facilement éliminées.

Nous eroyons done que la présure du chardon peut remplacer la présure animale, sans aucun désavantage, dans l'élaboration de la plupart des espèces de fromages connus dans notre pays.

\section{BIBLIOGRAPHIE}

[1] C. Christen et E. Virasoro. La loi d'action de la présure dans la coagulation du complexe caśinate de calcium + phosphate de calcium, Le Lait, $1932, \mathrm{p}, 923$.

[2] - - - 1932, p. 927 .

(1) Nos remerciments à Messieurs Chaunorge de la Fromagerie A. Bernard pour l'efficace collaboration qu'ils ont birn ı oulu nous prêter lors de ces essais. 
[3] J. Effront. Les catalyseurs biochimiques dans la vie et dans l'industrie, p. 96.

[4] - - , p. 97 .

[5] - - , p. 93.

[6] P. Partsi. Ricerche sulla "Cynarasi", Annali Istituto Spallanzani, vol. I, 1930, p. 147.

[7] C. Christen et E. Virasoro, Loc. cit.

[8] Haldane. Enzyme, p. 70.

$[9]-,-, p, 71$.

\title{
LA STABILISATION DU LAIT PAR LA DÉSACIDIFICATION ÉLECTRIQUE
}

\author{
par le Proíesseur Dr W. WINKLER (Vienne).
}

Le Lait a décrit, dans son numéro 132, de février 1934, un nouveau procédé qui permet d'une façon très simple, de réduire le degré d'acidité du lait par le courant électrique. Ce procédé permet de restituer à un lait, titrant $25^{\circ}$ Dornic d'acidité, la faculté, qu'il avait perdue, de pouvoir être bouilli et pasteurisé. Ceci présente un intérêt primordial également pour l'utilisation de la crème hyperacide dans la fabrication du beurre.

Ce procédé a trouvé son application industrielle dans les appareils de désacidification électrique construits par la Société "Elact" à Vienne.

L'article précité signalait đéjà que le traitement indiqué réduit la teneur du lait en bactéries et en germes. Les colibacilles nocifs se trouvant beaucoup plus atteints que les bactéries de l'acide lactique, on constate done une amélioration de la flore bactérienne du lait. La réduction de la teneur en germes implique, cela va de soi, un accroissement de la stabilité du lait, une prolongation de la durée de sa faculté d'ébullition.

Si le lait désacidifié est maintenu à basse température, entre 7 et $8^{\circ} \mathrm{C}$, , on augmente de 12 à 24 heures au moins - suivant le degré d'acidité originel - le temps pendant lequel le lait conserve sa faculté d'ébullition et de pasteurisation. A des températures plus élevées, à $20^{\circ}$ par exemple, l'augmentation de la stabilité est moindre, mais elle atteint toujours quelques heures au moins. Il faut, toutefois, que le lait traité ne titre pas plus de $38^{\circ}$ Dornic et que la désacidifieation soit poussée suffisamment loin.

Après le traitement, l'acidité reprend, d'abord, très lentement; elle monte, au contraire, très rapidement par la suite, ce qui s'explique par le fait que le procédé provoque la prépondérance, dans la flore, des bactéries de l'acide lactique.

L'accroissement de la stabilité est beaucoup plus important quand, après la désacidification, le lait est pasteurisé. On constate 